\section{Stitching together a song memory}

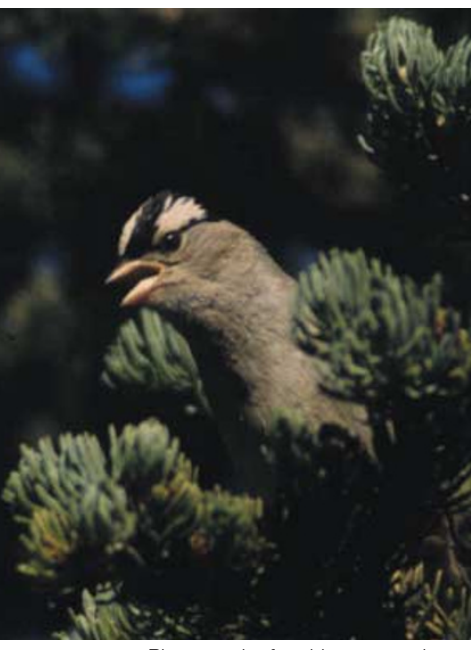

Photograph of a white-crowned sparrow, courtesy of F. Goller, University of Utah, USA.
New results published in Nature show that songbirds can accomplish remarkable feats of learning, at least when it comes to learning to sing. Rather like sequencing a genome by shotgun sequencing - splitting the genome into fragments that can be easily sequenced and then put back in the right order — white-crowned sparrows can learn their songs in pieces.

Shotgun sequencing requires a computer to reassemble the short sequences into the correct order by matching overlapping segments. Rose et al. show that white-crowned sparrows are capable of a similar feat when it comes to learning their song: after being exposed only to short but overlapping snippets of a 'tutor song', the young songbirds can reproduce the full tune. Beyond the remarkable animal behaviour story, the results offer insight into how the brain might store auditory memories that guide learned vocal behaviour.

The authors collected nestlings and exposed them to a recorded tutor song from the age of about 10 days. The researchers deliberately fragmented the recordings, so instead of a usual white-crowned song - which consists of four or five ordered 'phrases' of whistles, trills, buzzes and so on - the young birds listened to pairs of normally adjacent phrases. For example, if a full song consists of $\mathrm{ABCDE}$ (with each letter representing a phrase), the birds might have heard $\mathrm{DE}, \mathrm{CD}, \mathrm{BC}$ and $\mathrm{AB}$, with silent intervals separating the phrase pairs.

Rose et al. found that birds tutored on only adjacent phrase pairs could produce a normal, correctly ordered song, akin to singing 'Mary had a little lamb' correctly after only having ever heard 'little lamb' 'a little' 'had a' and 'Mary had'. Furthermore, in a separate experiment in which birds were tutored with reverse-order syllables (BA, $\mathrm{CB}$, etc.) the birds learned to sing a fully reversed song (EDCBA). This latter result is even more remarkable in light of previous evidence that white-crowned sparrows have a strong innate tendency - even in the absence of any tutor - to start songs with 'A-like' introductory phrases. The authors confirmed that the overlapping phrase-pairs were essential, as birds that only heard single phrases even though they heard all the phrases — could not sing normal songs.
ION CHANNELS

\section{Sensing the pressure}

Mechanosensitive ion channels convert external mechanical forces into electrical and chemical signals in the cell. In the case of the TREK-1 channel, it is opened by membrane stretch and cell swelling, which results in outward rectifying potassium currents and shaping of the action potential. Reporting in the EMBO Journal, Chemin and colleagues found that phospholipids are important in this mechanical gating process.

An artificial increase in the cytosolic concentration of phosphatidylinositol 4,5-bisphosphate ( PIP $\left._{2}\right)$ - a component of membrane phospholipids - stimulates the activity of endogenous TREK-1 in cultured striatal neurons. This elevation in channel activity is also seen when the cytosol is acidified, and the effects of both PIP $_{2}$ and low intracellular $\mathrm{pH}$ can be inhibited by the presence of positively charged intracellular molecules such as polylysine. The inhibitory effect of polylysine molecules is thought to be due to their interaction with the negative charges of membrane phospholipids, which prevents them from interacting electrostatically with specific positively charged segments in the channel protein.

The authors also show that PIP and acid can regulate the gating of TREK-1 in a cooperative manner: when TREK-1 is challenged with acid after addition of PIP $_{2}$, it becomes 'locked' in a highly active form even after the cytosolic $\mathrm{pH}$ is returned to $\mathrm{pH}$ 7.2. The activity of the locked TREK-1 channel is high even at atmospheric pressure, and pressure stimulation produces only a modest further activating effect. TREK-1 activation becomes more resistant to membrane stretch in the presence of polylysine, which indicates that the cationic molecule interferes with the pressure-sensing process.

Which segment of the channel is responsible for $\mathrm{PIP}_{2}$ stimulation? The authors generated truncated, chimaeric and point mutations in TREK-1, and analysed the channel activity in cells that overexpressed the mutant proteins. They found that a protein segment of the cytosolic carboxy-terminal domain of TREK-1 is crucial for $\mathrm{PIP}_{2}$ stimulation. This region contains several positively charged resides that seem to be attractive candidates for binding to phospholipids. A systematic substitution of the positively charged amino acids by alanines, which are neutral, progressively leads to reduced TREK-1 stimulation by $\mathrm{PIP}_{2}$. The authors conclude that the cationic cluster is important for stimulation of TREK-1 by phospholipids.
Chemin and colleagues then reasoned that if this positively charged region of TREK-1 is to interact with phospholipids, it must be localized near the plasma membrane. To show this, they used a complementation yeast system, in which a mutant strain fails to grow at the non-permissive temperature of $37^{\circ} \mathrm{C}$ unless it is rescued by the exogenously expressed amino-terminal fragment of hSOS, which lacks the membrane-targeting activity. As this fragment of hSOS is only functional when it gets to the plasma membrane, mutant yeast cells are not rescued unless hSOS is fused to another protein or protein segment that interacts with the plasma membrane. The authors show that the cytosolic cationic cluster of TREK-1 brought the hSOS fragment to the plasma membrane and allowed cell growth at $37^{\circ} \mathrm{C}$.

These findings have shed new light on the gating mechanism of the TREK channels, and might also have more general implications for other types of mechanically activated ion channel, as Hirono and colleagues recently suggested for the hair cell channel.

Jane Qiu

(0) References and links ORIGINAL RESEARCH PAPER Chemin, J. et al. A phospholipid sensor controls mechanogating of the $\mathrm{K}^{+}$ channel TREK-1. EMBO J. 2 December 2004 (10.1038/sj.emboj.7600494)

FURTHER READING Hirono. M. et al. Hair cells require phosphatidylinosital 4,5-bisphosphate for mechanical transduction and adaption. Neuron 44, 309-320 (2004) 